\title{
The Impact of Specialized Telephonic Guides on Employee Engagement in Corporate Well-Being Programs
}

\author{
Nicholas L. Boerger, MS, Nathan A. Barleen, BS, Mary L. Marzec, PhD, \\ Daniel P. Moloney, PhD, and Jeff Dobro, MD
}

\begin{abstract}
Employer-sponsored well-being programs have been growing in popularity as a means to control rising health care costs and increase workplace productivity. Engagement by employees is necessary for these programs to achieve their desired effects. Extrinsic motivators in the form of incentives and surcharges are commonly introduced by employer program sponsors to promote meaningful engagement. Although these may be successful in achieving a degree of engagement, individuals benefit by being intrinsically motivated as they modify behaviors and improve short- and long-term well-being. Telephonic guides equipped with motivational interviewing and other behavioral strategies to improve engagement may bridge the gap between extrinsic and intrinsic motivation. The objectives of this study are to determine characteristics associated with employee utilization of these guides when offered and to compare subsequent program engagement rates between utilizers to a propensity score matched group of employees who were not offered the service. The data were retrieved from a well-being program provider's database. The study examined 166,258 employees across 35 employers. It found utilizers were older, proportionally more female, in the manufacturing industry, incented to use the guide service, offered a larger incentive for program participation, had healthier self-reported behaviors, and had a higher perception of their employer's focus on well-being. The study found that guide utilizers were significantly more likely to engage in telephonic coaching, digital coaching, and activity tracking up to 6 months. The study's findings suggest telephonic guides using a range of behavioral techniques are an effective strategy to drive well-being program engagement.
\end{abstract}

Keywords: employer-sponsored, telephonic guide, program participation

\section{Introduction}

A GROWING NUMBER of large employers in the United States have adopted a well-being or health promotion program as a component of their employee health benefit. ${ }^{1,2}$ Although these programs are aimed at helping individuals improve their health status in myriad ways, including placing an increased value on health, setting well-being-related goals, participating in specific activities, and promoting social connectivity $^{3,4}$ to improve specific health risks, the study team and others recognize that most individuals will infrequently spontaneously change their health-related behaviors. Without meaningful engagement by employees, programs cannot achieve their desired effect. Variance in observed participation rates has prompted a vein of research dedicated to understanding what characteristics determine individual engagement. ${ }^{5}$ Although individual characteristics (eg, age, sex, education level) associated with participation are out of an employer's hands, there are strategies that may be employed to boost engagement rates.

To stimulate engagement, employers often introduce extrinsic motivators, in the form of incentives (direct payments, health plan premium reductions, drawing entries) and/or penalties (surcharges). ${ }^{6}$ These extrinsic motivators do not necessarily guarantee long-term success in molding positive behaviors. ${ }^{7} 8$ With a lack of intrinsic motivation, participation in well-being programs and achievement of long-term health status improvement may simply be seen by individuals as a

Department of Health Strategy, RedBrick Health, Minneapolis, Minnesota.

(C) Nicholas L. Boerger et al. 2018; Published by Mary Ann Liebert, Inc. This is an Open Access article distributed under the terms of the Creative Commons Attribution License, which permits unrestricted use, distribution, and reproduction in any medium, provided the original work is properly cited. 
task to be completed to earn an incentive or avoid a penalty. Therefore, successful well-being initiatives must effectuate the transition of those who initially engage as a result of extrinsic motivators to an intrinsically motivated state. ${ }^{9}$

A successful approach often benefits from an individual exploring opportunities for change and making a commitment to another human being as manifested in the technique of motivational interviewing (MI). ${ }^{10-12}$ MI leverages an individual's innate proclivity to regulate his or her behavior to grow and integrate in pursuit of personal well-being. ${ }^{13} \mathrm{MI}$ is utilized in the context of collaborative guided conversation with an individual to elicit personal goals, listening for and reinforcing "change talk," informing about relevant options, and closing a conversation with a commitment to make even a small change. ${ }^{12,14,15}$ Successful MI leads to an intrinsically motivated individual more able to successfully take specific action to change their behavior. ${ }^{16}$

There are additional techniques and strategies that can be employed within the context of the motivational interview to promote an individual's successful behavior change. The principles of ethical influence capture the effects of personal, social, and environmental impact on behavior. ${ }^{17-20}$ Habit formation techniques ${ }^{21}$ have been incorporated in many initiatives designed to improve successful adoption of a wide range of behaviors. The cognitive behavioral model, promotion of self-efficacy, mental contrasting techniques, ${ }^{22-25}$ and incorporation of the transtheoretical model ${ }^{26-29}$ have been demonstrated to improve the likelihood of success in making otherwise difficult behavior changes, such as increasing physical activity, eating healthier, and quitting smoking. ${ }^{30-32}$ Mental contrasting consists of soliciting one to envision the desired future state and contrast that with current reality along with overcoming obstacles for achieving the future state. ${ }^{33}$ Transtheoretical model-based consultation involves establishing the current stage of the individual, "precontemplation" through "maintenance," and consulting them based on where they are along the continuum. Scholars agree that a combination of techniques is ideal for successful interventions. $^{31}$

This study evaluates of the impact of a 1-time, short telephonic engagement support consultation with a guide ("Next Step Consult" [NSC]), based on a multi-foundational behavioral framework inclusive of the aforementioned principles and models. The primary goal of the consultation is to improve engagement in employer-sponsored health and wellbeing programs. The objectives of this paper are 2-fold. First, to determine individual, employer, and incentive characteristics associated with utilization of the consult service when offered. Second, to determine the short- and long-term effects of the consult service on participation in 3 types of well-being programs: telephonic coaching, digital coaching, and activity tracking.

\section{Methods}

\section{Intervention: NSC}

The telephonic engagement support session is referred to as "Next Step Consult" (NSC) as it is intended to help individuals take the next step to participate in well-being programs to improve their health after completing a health risk assessment (HRA) questionnaire or a biometric screening. Following completion of an HRA or biometric screening, individuals are able to schedule the NSC with a health guide trained in the aforementioned behavioral methods via a web portal or over the phone. The average NSC lasts 10 to 15 minutes.

During the consultation, the trained guide uses elements from the aforementioned behavioral change techniques appropriate for each individual to encourage him or her to commit to health improvement goals and engage in supportive well-being programs. The guide $(a)$ ensures that the individual understands their personal health risks (obtained from HRA and/or biometric screening), (b) assists the individual in identifying and understanding underlying personal motivators, $(c)$ facilitates the individual setting relevant health goals and breaking these into smaller and more easily achieved steps, and then $(d)$ encourages enrollment in appropriate well-being programs chosen by the individual.

Individuals included in this study were presented an online privacy policy through the wellness portal that describes the measures in place to protect their Personal Health Information and the use of data in accordance with the Privacy Rule of the Health Insurance Portability and Accountability Act of 1996 and employer-specific agreements. All data were de-identified prior to attainment by researchers for analysis.

\section{Setting}

This is a longitudinal observational study across 35 selfinsured employers who were contracted with a single wellbeing program provider as of January 1, 2014. Typically, decisions regarding well-being program offerings are made in collaboration between the employer, the well-being program provider, and third-party benefit consultants. Employers may offer different programs to subpopulations defined by individual characteristics such as relation to employee, benefit enrollment status, and other occupational characteristics such as whether an employee is full time. Available well-being programs were promoted on a personalized web portal. Following completion of an HRA and often a biometric screening, individuals were presented a series of recommended well-being programs and also were able to explore other available programs. When offered, NSC was presented at this point. Email communications encouraging use of NSC and well-being programs were frequently used to encourage participation. Additionally, print mailings, workplace signage or flyers, and portal notifications were used to promote wellbeing program participation.

\section{Inclusion criteria and outcomes}

Inclusion criteria for employees studied were: being continuously eligible throughout the employer-specific benefit season (1 year), completing an HRA, being offered all 3 wellbeing program modalities (telephonic coaching, digital coaching, and activity tracking), and having data available for all control variables of interest.

For the objective of determining characteristics associated with NSC utilization, the outcome of interest was whether an employee completed a call during their employer's specific benefit season. Employees not offered NSC were excluded from this analysis.

For the objective of estimating the effect of NSC call completion on longitudinal participation, the outcomes of interest were engagement in telephonic coaching, digital 
coaching, and activity tracking at 1 month, 3 months, and 6 months follow-up. Employees who were offered NSC and did not utilize it were excluded from this analysis to limit selection bias from the control sample. For the group of employees who completed a NSC call, the study team observed the month the call was completed and assigned that as their reference month. For the control group, comprised of employees not offered NSC, the team used the month of HRA completion as their reference month. Follow-up engagement months were then defined as the first, third, and sixth month following each individual's reference month. Participation definitions for each modality were: active enrollment in a telephonic coaching program, progressing in a digital coaching program, and logging activity (manually or using a sync device) during the respective follow-up month.

Telephonic coaching involves a series of scheduled calls with a well-being professional to set goals and improve health status across a spectrum of well-being domains. Enrollment in telephonic coaching begins following the completion of an initial assessment call, which is distinct from NSC, and continues with follow-up calls throughout the course of enrollment. Digital coaching programs are online, self-driven, nonhuman interactions allowing individuals to choose from a variety of steps or tasks to commit to and complete within a specific well-being focus area. Focus areas include tobacco cessation, nutrition, physical activity, weight loss, financial management, stress management, and disease management, among others. Activity tracking involves logging physical activity, either manually online or using a synced electronic device such as a pedometer, to allow individuals to monitor and evaluate self-advancement toward wellness goals.

\section{Control variables}

Age and sex were retrieved from personnel files received from employers. Incentive structure documentation from the well-being program provider included incentive amounts for utilization of NSC as well as for participation in well-being programs. Employer industry was derived using the North American Industry Classification System. ${ }^{34}$ Of those incented to utilize NSC, $75.0 \%$ were offered $\$ 50$ and the rest were offered some amount less than that. Given lack of variability in the amount incented, the study team dichotomized individuals as incented or not. Variability in program participation amounts (total available for telephonic coaching, digital coaching, and activity tracker) was limited as well. Amounts were categorized at \$0, \$1-\$399, and \$400+.

Derived from the HRA were perceived employer support of health (culture) and composite health score. The 5 response categories describing perceived employer focus on employee health ranged from "[employer] is not focused on employee health and has work practices that are actually bad for health" to "[employer] actively promotes the health of employees." Responses concentrated on the latter $(62.7 \%$ of responses). Responses were dichotomized so that responding in the highest category was labeled "high" and the other 4 were combined into "low." The composite health score was continuous and based on weighted responses to questions across a multitude of domains including tobacco exposure, cardiovascular health, stress and coping, nutrition, physical activity, among others. Higher scores represented preferred health behaviors and favorable biometrics. As the health score was highly left-skewed, quartiles were applied to categorize responses across the continuous spectrum.

Other relation types (eg, spouses, adult dependents) were excluded from the analysis for multiple reasons. The study team did not know if and where nonemployees were employed and could not prescribe their workplaces' industries. The perceived employer focus on employee health question from the HRA could potentially be interpreted by nonemployees in a number of ways: in regard to the employee's employer, their own employer, or not applicable if they are unemployed. The overall experience of nonemployees differs from that of employees in intangible ways that make their inclusion in this analysis untenable.

\section{Statistical analysis}

Three statistical tests were employed throughout the course of this research. Chi-square testing was used to compare covariate differences between 2 sets of study cohorts: employees who were offered and utilized NSC versus those who were offered and did not utilize (describing who uses this service when offered), and employees who were offered and utilized NSC versus those who were not offered (before and after matching, to answer whether the groups used in propensity score matching differed systematically). A probit model regression was run on the former cohort to determine characteristics associated with completion of a NSC call when offered. Finally, a propensity score matching technique was employed to estimate the effects of NSC call completion on longitudinal well-being program participation. All analyses were run using Stata version 13 (StataCorp, College Station, TX). ${ }^{35}$

Individuals who were not offered NSC (control cohort) were matched without replacement using propensity scores to individuals who completed a NSC call (treated cohort). Average treatment effects on the treated (ATT) were estimated across each of the 3 program modalities at each of the follow-up months.

\section{Results}

A total population of 166,258 employees met the criteria for inclusion in the study. Within the total population, $60,506(36.4 \%)$ were eligible to utilize NSC based on their product offering. Of those eligible, 11,302 (18.7\%) utilized the service.

Descriptive statistics of the study sample are shown in Table 1. All covariates were significantly different. NSC utilizers were proportionally younger and more were male. The proportion who were incented to utilize NSC was greater and they distributed toward the higher participation incentive categories. Utilizers were more likely to be in the manufacturing industry, distributed more heavily into higher (healthier) composite health score quartiles, and more likely to respond to the highest perceived employer support category.

\section{Characteristics of NSC call completers}

Table 2 shows the predictive margins by covariate from the probit regression analysis.

After controlling for other covariates, females were significantly were more likely to utilize NSC than males 
Table 1. Descriptive Statistics for Study Cohorts $(N=166,258)$

\begin{tabular}{|c|c|c|c|c|c|c|c|}
\hline \multirow[b]{2}{*}{ Covariate } & \multirow[b]{2}{*}{ Group } & \multirow[b]{2}{*}{$\begin{array}{c}\text { Total population } \\
(\mathrm{n}=166,258) \\
\mathrm{n}(\%)\end{array}$} & \multicolumn{3}{|c|}{ NSC offered } & \multicolumn{2}{|c|}{ NSC not offered } \\
\hline & & & $\begin{array}{c}\text { Utilized } \\
(\mathrm{n}=11,302), \\
\mathrm{n}(\%)\end{array}$ & $\begin{array}{c}\text { Not utilized } \\
(\mathrm{n}=49,204) \\
\mathrm{n}(\%)\end{array}$ & $\mathrm{P}^{\mathrm{a}}$ & $\begin{array}{c}\text { Not utilized } \\
(\mathrm{n}=105,752), \\
\mathrm{n}(\%)\end{array}$ & $\mathrm{P}^{\mathrm{b}}$ \\
\hline \multirow[t]{4}{*}{ Age group } & $18-29$ & $25,365(15.3)$ & $1584(14.0)$ & 7838 (15.9) & \multirow[t]{4}{*}{$<0.001$} & $15,943(15.1)$ & \multirow[t]{4}{*}{$<0.001$} \\
\hline & $30-39$ & $48,391(29.1)$ & $3565(31.5)$ & $14,924(30.3)$ & & $29,902(28.3)$ & \\
\hline & $40-49$ & $48,154(29.0)$ & $3658(32.4)$ & $14,551(29.6)$ & & $29,945(28.3)$ & \\
\hline & $50-64$ & $44,348(26.7)$ & $2495(22.1)$ & $11,891(24.2)$ & & $29,962(28.3)$ & \\
\hline Female & & $80,458(48.4)$ & 4365 (38.6) & $21,253(43.2)$ & $<0.001$ & $54,840(51.9)$ & $<0.001$ \\
\hline NSC incented & & $45,779(27.5)$ & $9738(86.2)$ & $36,041(73.3)$ & $<0.001$ & N/A & - \\
\hline \multirow{3}{*}{$\begin{array}{l}\text { Participation } \\
\text { incentive }\end{array}$} & $\$ 0$ & $12,262(7.4)$ & $425(3.8)$ & $3551(7.2)$ & \multirow[t]{3}{*}{$<0.001$} & $8286(7.8)$ & \multirow[t]{3}{*}{$<0.001$} \\
\hline & $\$ 1-\$ 399$ & $124,527(74.9)$ & $6761(59.8)$ & $38,298(77.8)$ & & $79,468(75.2)$ & \\
\hline & $\$ 400+$ & 29,469 (17.7) & $4116(36.4)$ & 7355 (15.0) & & $17,998(17.0)$ & \\
\hline \multirow[t]{3}{*}{ Industry } & Manufacturing & $48,176(29.0)$ & 6095 (53.9) & $19,138(38.9)$ & \multirow[t]{3}{*}{$<0.001$} & $22,943(21.7)$ & \multirow[t]{3}{*}{$<0.001$} \\
\hline & Professional & $95,078(57.2)$ & $4628(41.0)$ & $20,284(41.2)$ & & $70,166(66.4)$ & \\
\hline & Service & $23,004(13.8)$ & $579(5.1)$ & 9782 (19.9) & & $12,643(12.0)$ & \\
\hline \multirow{4}{*}{$\begin{array}{l}\text { HRA health } \\
\text { score }\end{array}$} & Quartile 1 & $41,559(25.0)$ & $1956(17.3)$ & $10,494(21.3)$ & \multirow[t]{4}{*}{$<0.001$} & $29,109(27.5)$ & \multirow[t]{4}{*}{$<0.001$} \\
\hline & Quartile 2 & $41,520(25.0)$ & $2647(23.4)$ & $11,909(24.2)$ & & $26,964(25.5)$ & \\
\hline & Quartile 3 & $41,569(25.0)$ & $3071(27.2)$ & $13,044(26.5)$ & & $25,454(24.1)$ & \\
\hline & Quartile 4 & $41,610(25.0)$ & $3628(32.1)$ & $13,757(28.0)$ & & 24,225 (22.9) & \\
\hline \multirow[t]{2}{*}{ HRA culture } & Low & $61,940(37.26)$ & $3671(32.5)$ & $17,241(35.0)$ & \multirow[t]{2}{*}{$<0.001$} & $41,028(38.8)$ & \multirow[t]{2}{*}{$<0.001$} \\
\hline & High & $166,258(62.7)$ & $7631(67.5)$ & $31,963(65.0)$ & & $64,724(61.2)$ & \\
\hline
\end{tabular}

${ }^{\text {a }} P$-values for $\chi_{2}^{2}$ testing differences in covariates between individuals offered NSC who utilized it to those who did not utilize it. ${ }^{\mathrm{b}} P$-values for $\chi^{2}$ testing differences in covariates between individuals offered NSC who utilized it to those not offered NSC.

HRA, health risk assessment; NSC, Next Step Consult.

(Table 2). Using the youngest age group (18-29 years old) as reference, all other age groups were significantly more likely to utilize NSC. Utilization of NSC was 3.5 times higher for employees offered an incentive to use it than those not incented. Employees with incentives tied to participation in the program modalities were significantly more likely to utilize NSC. Employees in the manufacturing in-

TABle 2. Margins by Covariate

\begin{tabular}{lll}
\hline Covariate & \multicolumn{1}{c}{ Group } & Margin \\
\hline Age group & $18-29$ (Ref) & 0.146 \\
& $30-39$ & $0.168^{\mathrm{a}}$ \\
& $40-49$ & $0.168^{\mathrm{a}}$ \\
Sex & $50-64$ & $0.171^{\mathrm{a}}$ \\
& Male (Ref) & 0.153 \\
NSC incented & Female & $0.182^{\mathrm{a}}$ \\
Participation incentive & No (Ref) & 0.060 \\
& Yes (Ref) & $0.215^{\mathrm{a}}$ \\
Industry & \$1-\$399 & 0.130 \\
& \$400+ & $0.157^{\mathrm{a}}$ \\
HRA health score & Manufacturing (Ref) & $0.211^{\mathrm{a}}$ \\
& Professional & 0.275 \\
& Service & $0.155^{\mathrm{a}}$ \\
& Quartile 1 (Ref) & $0.037^{\mathrm{a}}$ \\
& Quartile 2 & 0.148 \\
& Quartile 3 & $0.161^{\mathrm{a}}$ \\
HRA culture & Quartile 4 & $0.164^{\mathrm{a}}$ \\
& Low (Ref) & $0.182^{\mathrm{a}}$ \\
& High & 0.156 \\
\end{tabular}

a Significant at $P<0.05$.

HRA, health risk assessment; NSC, Next Step Consult. dustry had a significantly greater likelihood of utilizing than the professional and service industries. Relative to the lowest HRA score quartile each of the other quartiles had a significantly greater likelihood of utilizing NSC. Employees perceiving their employer to be highly focused on their health were significantly more likely to utilize (Table 2).

\section{Estimated effects of NSC on longitudinal participation}

There were 11,302 eligible employees who were offered and utilized NSC. A cohort of 11,302 controls was then matched using a 1:1 non-replacement propensity score matching technique. Table 3 offers a comparison of covariates between NSC utilizers and controls after matching. None are statistically different.

Table 4 offers the ATT for each outcome of interest post match. Participation rates across all program modalities were significantly greater for individuals in the NSC cohort. All were statistically significant.

For both cohorts, telephonic coaching enrollment increased between 1 month and 3 months and then decreased at 6 months (Table 4). The other 2 modalities decreased over time from 1 month through 6 months for both cohorts. Active engagement in digital coaching tapered $15.4 \%$ for NSC utilizers and $4.1 \%$ for nonutilizers over the time period between 1 and 6 months. Active engagement in activity tracking tapered $11 \%$ for NSC utilizers and .9\% for nonutilizers over the same time period. In all instances rate ratios decreased over time. To provide context for these engagement rates, the study team observed $13,485(59.7 \%)$ of the total matched cohort engaged in at least 1 of the program modalities in the 6 months following their reference month. 
Table 3. Matched SAmples Comparison $(N=22,604)$

\begin{tabular}{|c|c|c|c|c|}
\hline \multirow[b]{2}{*}{ Covariate } & \multirow[b]{2}{*}{ Group } & \multicolumn{3}{|c|}{ Cohorts } \\
\hline & & Utilized $(\mathrm{n}=11,302), \mathrm{n}(\%)$ & Not offered $(\mathrm{n}=11,302), \mathrm{n}(\%)$ & $\mathrm{P}$ \\
\hline \multirow[t]{4}{*}{ Age group } & $18-29$ & $1584(14.0)$ & 1587 (14.0) & 1.0 \\
\hline & $30-39$ & 3565 (31.5) & 3561 (31.5) & \\
\hline & $40-49$ & 3658 (32.4) & 3658 (32.4) & \\
\hline & $50-64$ & 2495 (22.1) & $2496(22.1)$ & \\
\hline Female & & 4365 (38.6) & 4355 (38.5) & 0.891 \\
\hline \multirow[t]{3}{*}{ Participation incentive } & $\$ 0$ & $425(3.8)$ & $424(3.8)$ & 0.999 \\
\hline & $\$ 1-\$ 399$ & $6761(59.8)$ & $6760(59.8)$ & \\
\hline & $\$ 400+$ & $4116(36.4)$ & 4118 (36.4) & \\
\hline \multirow[t]{3}{*}{ Industry } & Manufacturing & 6095 (53.9) & 6095 (53.9) & 1.0 \\
\hline & Professional & $4628(41.0)$ & $4628(41.0)$ & \\
\hline & Service & $579(5.1)$ & $579(5.1)$ & \\
\hline \multirow{4}{*}{ HRA health score } & Quartile 1 & 1956 (17.3) & 1958 (17.3) & 1.0 \\
\hline & Quartile 2 & 2647 (23.4) & 2646 (23.4) & \\
\hline & Quartile 3 & $3071(27.2)$ & $3064(27.1)$ & \\
\hline & Quartile 4 & $3628(32.1)$ & 3634 (32.2) & \\
\hline \multirow[t]{2}{*}{ HRA culture } & Low & $3671(32.5)$ & $33,673(32.5)$ & 0.977 \\
\hline & High & $7631(67.5)$ & 7629 (67.5) & \\
\hline
\end{tabular}

HRA, health risk assessment.

\section{Discussion}

The purpose of this research was to identify characteristics of NSC utilizers compared to nonutilizers and to estimate the effect of NSC utilization on engagement in well-being programs.

\section{Characteristics of NSC call completers}

This study presents a significant gender effect in NSC utilization. Previous research has found females participate in well-being programs at higher rates than men, ${ }^{36}$ a finding observed in the present study. In studying workplace wellness programs of Fortune 100 employers, Mattke et al found females were more likely to complete an HRA but men were more likely to participate in lifestyle management and disease management programs. ${ }^{2}$ NSC occurs after the HRA in this study and would be somewhat comparable to a lifestyle or disease management program in terms of individual involvement, although it is a single interaction rather than an ongoing program. The influence of sex on well-being is complex and would benefit from further investigation.
The youngest age group was least likely to utilize NSC. In other studies it has been generally observed that older age groups demonstrated an increased willingness to utilize well-being program opportunities. ${ }^{2,36,37}$ Aging is associated with increased condition prevalence, health care expenditures, and poorer health status such as obesity, ${ }^{38}$ which may be cause for increased health awareness and willingness to engage in opportunities to improve health. Similar to sex, age has a complex relationship with if and how an individual engages in their well-being.

The way programs are incented affects whether or not an individual chooses to engage in them, especially if the individual is not intrinsically motivated. Having a direct financial incentive tied to NSC was associated with increased utilization. Having incentives tied to program participation also was associated with greater utilization of NSC. A greater overall incentive offering may be indicative of an overall stronger well-being promotion culture at a given employer. Previous literature has concluded that insufficient incentives are a barrier to well-being program participation. ${ }^{39}$ Effectiveness of incentives may be assessed a variety of ways. In the case of a telephonic guide service, incentive effectiveness may be

Table 4. Average Treatment Effect on Treated

\begin{tabular}{|c|c|c|c|c|c|c|c|}
\hline Program modality & Time & Treated, \% & Controls, \% & Diff., \% & Rate ratio & $S E$ & $t$ Test $^{\mathrm{a}}$ \\
\hline Telephonic coaching & 1 Month & 15.7 & 4.0 & 11.7 & 3.9 & 0.004 & 30.20 \\
\hline Telephonic coaching & 3 Months & 17.0 & 4.8 & 12.2 & 3.5 & 0.004 & 29.93 \\
\hline Telephonic coaching & 6 Months & 13.1 & 4.7 & 8.4 & 2.8 & 0.004 & 22.30 \\
\hline Digital coaching & 1 Month & 28.5 & 10.4 & 18.1 & 2.7 & 0.005 & 35.19 \\
\hline Digital coaching & 3 Months & 22.2 & 8.5 & 13.7 & 2.6 & 0.005 & 29.10 \\
\hline Digital coaching & 6 Months & 13.1 & 6.3 & 6.8 & 2.1 & 0.004 & 17.28 \\
\hline Activity tracking & 1 Month & 52.3 & 21.6 & 30.7 & 2.4 & 0.006 & 50.39 \\
\hline Activity tracking & 3 Months & 48.8 & 21.0 & 27.8 & 2.3 & 0.006 & 45.84 \\
\hline Activity tracking & 6 Months & 41.3 & 20.7 & 20.6 & 2.0 & 0.006 & 34.23 \\
\hline
\end{tabular}

${ }^{a}$ All $t$ tests significant at $P<0.05$.

Diff., difference; SE, standard error. 
measured first by an increased likelihood of utilizing the service and, second, an increased likelihood of program participation after utilization of the service.

Work environment and varying job requirements by industry are important considerations when attempting to effectively engage a specific employee population. Others have noted that engagement in telephonic coaching varied by industry and that both employer support (physical environment and communications) and employee characteristics (female and being older) influenced program engagement and completion. ${ }^{40}$ In this study, the employees in the manufacturing industry were significantly more likely to utilize the guide service than both the professional and service industry employees. Employees in the manufacturing industry likely have less access to computers while at work, which may lead them to prefer telephonic engagement options over digital. ${ }^{40}$ There may be different cultural and interpersonal preference differences as well. This finding underscores the need for employers to offer well-being support through a variety of modalities to meet diverse needs across their employee population.

\section{Estimated effects of NSC on longitudinal participation}

Telephonic consultation with a guide was associated with a significant increase in short- and long-term engagement across all well-being program modalities. There were differences in relative rates for each of the modalities measured. Engagement rate ratios between NSC utilizers and nonutilizers were considerably smaller for digital coaching and activity tracking relative to phone coaching. This may be because NSC utilizers have an overall predisposition toward human interaction compared to digital interaction. Nonutilizers may be less interested in personal interactions regarding their health and may avoid NSC as well as phone coaching, preferring to engage in self-directed program options.

The most utilized program modality for both groups at all time frames was activity tracking. In addition to being useful as a stand-alone modality, activity tracking complements the other modalities as it allows an individual to easily evaluate his or her nutrition and physical activity behaviors, which are aspects of virtually every focus area in the telephonic and digital coaching modalities.

\section{Limitations}

Although this paper did show that NSC support resulted in higher rates of program participation, some limitations should be noted. One limitation is that the study focused solely on downstream well-being program engagement. Changes in health risks and other metrics such as health care claims were beyond the scope of this study. Follow-up research would be useful to document the impact of NSC on these measures as well.

For methods, the use of one-to-one nearest neighbor matching without replacement effectively reduced bias within observable variables and was possible because of a large data set with a majority of observations being controls (not offered service). However, it is appropriate to note that propensity score matching could not control for all differences between NSC utilizers and nonutilizers. Given the observational data underlying this study, unmeasured cov- ariates are not necessarily evenly distributed among groups. One can nonetheless reasonably assume that the present study findings are reliably controlling for observable extraneous differences between NSC utilizers and matched controls.

Lack of variance in incentive structures (eg, specific program modality amounts, NSC amounts, payout cadence) led to issues in collinearity and an inability to measure the specific effects of these characteristics. In addition to increasing confidence in the models, further research on the effects of incentive structures on NSC utilization as well as longitudinal participation would lend itself to strategic recommendations on resource allocation for optimizing employee engagement in well-being programs.

How well-being program opportunities were communicated by employers varied in frequency, medium (eg, workplace flyers, electronic communications), and may have been focused on certain segments of the employee population. Data points on communications were not readily available and represent a limitation of this study. This is another facet of workplace wellness deserving of research to increase engagement.

The phone coaching outcome used was active enrollment during the respective month. This measure was used because engagement in the phone coaching program was episodic and individual calls could occur multiple times per month but also were not necessarily occurring each month measured. Using call completion would have opened the possibility for an actively enrolled individual to be counted as not engaged in a given month depending on their call cadence. Phone enrollments were extended time frames and rather insensitive, at least relative to digital coaching programs and activity tracking. A discrete measure such as call completion would have been more aligned with the other modalities' definitions but would open the possibility of misclassification.

Given that employee health benefit seasons were 12 months, extending participation time frames past 6 months would increasingly open the possibility for overlap into the following season. In excess this overlap could obscure results because of additional exposure to reference interventions (HRAs and NSCs) and seasonal changes to the well-being program. Future research may investigate whether additional intervention continues to stimulate well-being program engagement and in what estimated dosing gradient.

\section{Conclusions}

\section{The impact of a behavior change model}

The well-being industry has long offered an introductory telephonic interaction (often referred to as health advisor) to present options for employees to engage in healthy activities within well-being programs. The study team has no available data regarding the impact of these traditional approaches but employers have noted consistently that these have had minimal impact on program engagement. The professional guides providing the NSC service studied had received specific training in a wide range of behavior change methods and most have prior credentials and training in areas of clinical lifestyle improvement such as registered dietitian, exercise physiology, social work, health education, and/or public health. The interaction model developed for 
this service incorporates a wide range of behavioral change methods, in combination with fundamental MI techniques to personalize the approach with each individual. This process takes advantage of the personal accountability to another human resulting from the commitments made during this conversation. The study's findings suggest telephonic guides using a range of behavioral techniques are an effective strategy to drive well-being program engagement.

\section{Author Disclosure Statement}

Drs. Marzec, Moloney, and Dobro, Mr. Boerger, and Mr. Barleen declared the following potential conflicts of interest with respect to the research, authorship, and/or publication of this article: At the time of analysis all authors were employed at RedBrick Health, the wellness provider from which data were obtained. The telephonic guide service researched in this study is a product offered by RedBrick Health that may have a fee associated. The interest of this paper is, however, to understand the impact of the guide service and its underlying tenets on engagement in corporate well-being programs. This work was supported through the authors' employment at RedBrick Health.

\section{References}

1. Baicker K, Cutler D, Song Z. Workplace wellness programs can generate savings. Health Aff (Millwood) 2010; 29:304-311.

2. Mattke S, Liu H, Caloyeras JP, Huang CY, Van Busum KR. Workplace wellness programs study: final report. Santa Monica, CA: Rand, 2013.

3. McSpadden KE, Patrick H, Oh AY, Yaroch AL, Dwyer LA, Nebeling LC. The association between motivation and fruit and vegetable intake: the moderating role of social support. Appetite 2016;96:87-94.

4. Asch DA, Rosin R. Engineering social incentives for health. N Engl J Med 2016;375:2511-2513.

5. Robroek SJ, van Lenthe FJ, van Empelen P, Burdorf A. Determinants of participation in worksite health promotion programmes: a systematic review. Int J Behav Nutr Phys Act 2009;6:26.

6. Schmidt H, Voigt K, Wikler D. Carrots, sticks, and health care reform-problems with wellness incentives. N Engl J Med 2010;362:e3.

7. Seifert CM, Chapman LS, Hart JK, Perez P. Enhancing intrinsic motivation in health promotion and wellness. Am J Health Promot 2012;26:TAHP1-TAHP12.

8. Gingerich SB, Anderson DR, Koland H. Impact of financial incentives on behavior change program participation and risk reduction in worksite health promotion. Am $\mathrm{J}$ Health Promot 2012;27:119-122.

9. Deci EL, Ryan RM. The "what" and "why" of goal pursuits: human needs and the self-determination of behavior. Psychol Inq 2000;11:227-268.

10. Linden A, Butterworth SW, Prochaska JO. Motivational interviewing-based health coaching as a chronic care intervention. J Eval Clin Pract 2010;16:166-174.

11. Martins RK, McNeil DW. Review of motivational interviewing in promoting health behaviors. Clin Psychol Rev 2009;29:283-293.

12. Rollnick S, Butler CC, Kinnersley P, Gregory J, Mash B. Motivational interviewing. BMJ 2010;340:c1900.
13. Ryan RM, Deci EL. Self-determination theory and the facilitation of intrinsic motivation, social development, and well-being. Am Psychol 2000;55:68-78.

14. Markland D, Ryan RM, Tobin VJ, Rollnick S. Motivational interviewing and self-determination theory. J Soc Clin Psychol 2005;24:811-831.

15. Resnicow K, McMaster F. Motivational interviewing: moving from why to how with autonomy support. Int $\mathbf{J}$ Behav Nutr Phys Act 2012;9:19.

16. Teixeira PJ, Carraca EV, Markland D, Silva MN, Ryan RM. Exercise, physical activity, and self-determination theory: a systematic review. Int $\mathbf{J}$ Behav Nutr Phys Act 2012;9:78.

17. Cialdini RB. Influence: science and practice, 5th edition. Boston: Pearson Education, 2009.

18. Jacobson RP, Mortensen CR, Cialdini RB. Bodies obliged and unbound: differentiated response tendencies for injunctive and descriptive social norms. J Pers Soc Psychol 2011;100:433-448.

19. Nolan JM, Schultz PW, Cialdini RB, Goldstein NJ, Griskevicius V. Normative social influence is underdetected. Pers Soc Psychol Bull 2008;34:913-923.

20. Cialdini RB, Goldstein NJ. Social influence: compliance and conformity. Annu Rev Psychol 2004;55:591-621.

21. Fogg BJ. A behavior model for persuasive design. In: Paper presented at: Proceedings of the 4th International Conference on Persuasive Technology, Claremont, CA, April 26-29, 2009.

22. Kappes A, Oettingen G. The emergence of goal pursuit: mental contrasting connects future and reality. J Exp Soc Psychol 2014;54:25-39.

23. Kappes A, Singmann H, Oettingen G. Mental contrasting instigates goal pursuit by linking obstacles of reality with instrumental behavior. J Exp Soc Psychol 2012;48:811818.

24. Loy LS, Wieber F, Gollwitzer PM, Oettingen G. Supporting sustainable food consumption: mental contrasting with implementation intentions (MCII) aligns intentions and behavior. Front Psychol 2016;7:607.

25. Sevincer AT, Oettingen G. Spontaneous mental contrasting and selective goal pursuit. Pers Soc Psychol Bull 2013;39: 1240-1254.

26. Prochaska JO, Velicer WF. The transtheoretical model of health behavior change. Am J Health Promot 1997;12: 38-48.

27. Greene GW, Redding CA, Prochaska JO, et al. Baseline transtheoretical and dietary behavioral predictors of dietary fat moderation over 12 and 24 months. Eat Behav 2013; $14: 255-262$.

28. Mastellos N, Gunn LH, Felix LM, Car J, Majeed A. Transtheoretical model stages of change for dietary and physical exercise modification in weight loss management for overweight and obese adults. Cochrane Database Syst Rev 2014;CD008066. DOI: 10.1002/14651858.CD008066 .pub3.

29. Norcross JC, Krebs PM, Prochaska JO. Stages of change. J Clin Psychol 2011;67:143-154.

30. Oettingen G, Mayer D, Thorpe J. Self-regulation of commitment to reduce cigarette consumption: mental contrasting of future with reality. Psychol Health 2010;25: 961-977.

31. Winter SJ, Sheats JL, King AC. The use of behavior change techniques and theory in technologies for cardiovascular 
disease prevention and treatment in adults: a comprehensive review. Prog Cardiovasc Dis 2016;58:605-612.

32. Johannessen KB, Oettingen G, Mayer D. Mental contrasting of a dieting wish improves self-reported health behaviour. Psychol Health 2012;27:43-58.

33. Achtziger A, Fehr T, Oettingen G, Gollwitzer PM, Rockstroh B. Strategies of intention formation are reflected in continuous MEG activity. Soc Neurosci 2009;4:11-27.

34. U.S. Census Bureau. North American Industry Classification System. 2012. www.census.gov/cgi-bin/sssd/naics/naicsrch Accessed August 1, 2016.

35. StatCorp. Stata Statistical Software: Release 13 [computer program]. College Station, TX: StataCorp LP, 2013.

36. Ovbiosa-Akinbosoye OE, Long DA. Wellness program satisfaction, sustained coaching participation, and achievement of health goals. J Occup Environ Med 2012;54:592-597.

37. Norman GJ, Heltemes KJ, Drew J. Examining incentive design strategies for worksite wellness program engagement. Popul Health Manag 2014;17:324-331.
38. Pitt-Catsouphes M, James JB, Matz-Costa C. Workplacebased health and wellness programs: the intersection of aging, work, and health. Gerontologist 2015;55:262-270.

39. Osilla KC, Van Busum K, Schnyer C, Larkin JW, Eibner C, Mattke S. Systematic review of the impact of worksite wellness programs. Am J Manag Care 2012;18:e68-e81.

40. Grossmeier J. The influence of worksite and employee variables on employee engagement in telephonic health coaching programs: a retrospective multivariate analysis. Am J Health Promot 2013;27:e69-e80.

Address correspondence to: Nicholas L. Boerger, MS

RedBrick Health

510 Marquette Avenue South

Suite 500

Minneapolis, MN 55402

E-mail: nboerger@redbrickhealth.com 